\title{
Artificial Neural Networks for Dairy Industry: A Review
}

\author{
Sumit Goyal and Gyanendra Kumar Goyal \\ National Dairy Research Institute, Karnal-132001, India \\ E-mail: thesumitgoyal@gmail.com,gkg5878@yahoo.com
}

\begin{abstract}
Artificial Neural Networks (ANNs) have been implemented in almost every field, viz., science, technology, medicine and engineering as they have proved useful tools for obtaining the desired output including the analyses and shelf life prediction in case of food products. This review discusses the systematic information available in the literature concerning the implementation of ANN models for shelf life determination of milk, various dairy products, viz., yoghurt, chocolate, butter, cheese, kalakand, dairy confections and milk based coffee drinks, which would be very useful for dairy industry, consumers, wholesalers, retailers, regulatory agencies, food researchers and academicians.
\end{abstract}

Keywords: Artificial Neural Network, Artificial Intelligence, Shelf Life Prediction, Dairy Products

\section{Introduction}

The application of artificial neural networks (ANNs) for predicting the shelf life of food products in food industry is quite a new and effective approach. The ANN technique to food products was applied mainly because the shelf life evaluation in the laboratory is very cumbersome, expensive, and time-consuming, while the ANN procedure is sensitive, reliable, fast, simple and low-cost method for monitoring the authenticity of the products which provide consumers with a safer food supply. ANNs have been implemented in almost every field of science and technology as they have proved useful tools for obtaining the desired output including the analyses and shelf life prediction in case of food products, which has been very beneficial for the food industry, consumers, wholesalers, retailers, regulatory agencies, food researchers and academicians. 
ANNs are inspired by the early models of sensory processing by the brain. An ANN can be created by simulating a network of model neurons in a computer. By applying algorithms that mimic the processes of real neurons, one can make the network 'learn' to solve many types of problems. A model neuron is referred to as a threshold unit. It receives input from a number of other units or external sources, weighs each input and adds them up. If the total input is above a threshold, the output of the unit is one; otherwise it is zero. Therefore, the output changes from 0 to 1 when the total weighted sum of inputs is equal to the threshold. The points in input space satisfying this condition define a so called hyperplane. In two dimensions, a hyperplane is a line, whereas in three dimensions, it is a normal plane. Points on one side of the hyperplane are classified as 0 and those on the other side as 1 . Thus, a classification problem can be solved by a threshold unit if the two classes can be separated by a hyperplane [1].

\subsection{Single Layer and Multilayer Networks}

Single layer perceptron network consists of a single layer of output nodes; the inputs are fed directly to the outputs via a series of weights. The sum of the products of the weights and the inputs is calculated in each node, and if the value is above some threshold (typically 0 ) the neuron fires and takes the activated value (typically 1 ); otherwise it takes the deactivated value (typically -1 ). Neurons with this kind of activation function are also called artificial neurons or linear threshold units, while the multilayer networks consists of multiple layers of computational units, usually interconnected in a feedforward way. Multilayer networks use a variety of learning techniques, the most popular being backpropagation, and the output values are compared with the correct answer to compute the value of some predefined error-function. By various techniques, the error is then fed back through the network. Using this information, the algorithm adjusts the weights of each connection in order to reduce the value of the error function by some small amount. After repeating this process for a sufficiently large number of training cycles, the network usually converge to some state where the error of the calculations is small [2]. The input and output training pattern of the ANN is illustrated in Fig.1. 


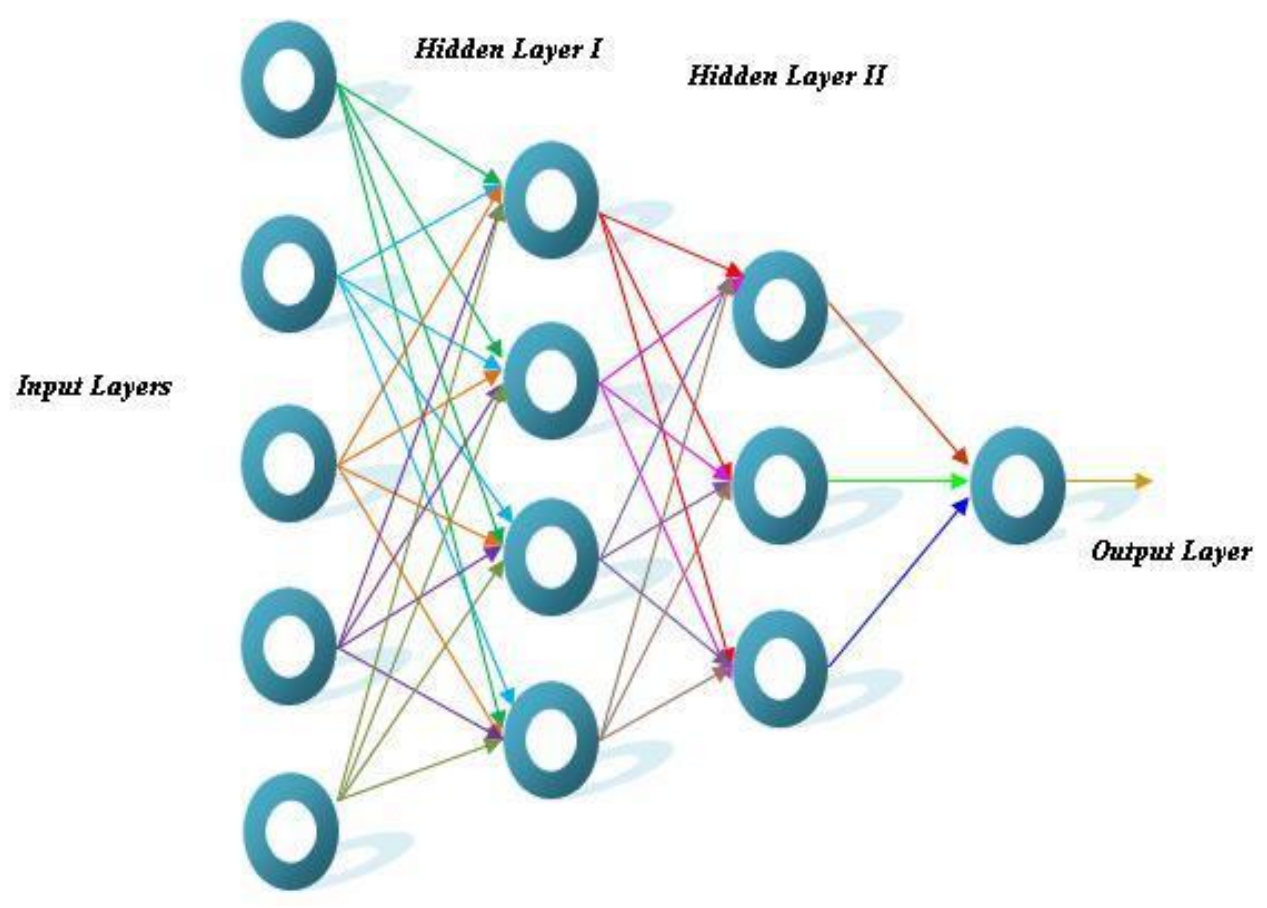

Figure1. Inputs and output layer of ANN models

\section{Shelf Life}

Shelf life studies provide important information to product manufacturers and developers to ensure that the consumer will continue to get a high quality product for a significant period of time after its production. Since the shelf life evaluation conducted in the laboratory is a long time-consuming process, and does not fit with the speed requirement of the food industry; therefore accelerated methods for estimating the shelf life of food products have been recently developed. The modern food industry has flourished because of its ability to deliver a wide variety of high quality food products to consumers. This has been achieved by building stability into the products through adopting various technological techniques like processing, packaging, and additives. Consumer demands for convenience have fueled new innovations in the food product development, packaging and chemical industries, and the widespread desire for products to use in the microwave oven has added further impetus to this effort. As an increasing number of new foods compete for getting space on supermarket shelves, the words "speed and innovation" have become the watchwords for food companies seeking to become "first to market" with successful products. Overall quality of the product is of prime importance in present day competitive market and needs to be built into the speed and innovation system. How the consumer feels about the product is the ultimate index of food quality. Therefore, the quality built in during the 
development of the product and production process must last through the distribution and consumption stages [3].

\section{ANNs for Prediction in Dairy Industry}

\subsection{Milk}

The usefulness of ANN models for prediction of shelf-life of milk by multivariate interpretation of gas chromatographic profiles, and flavour-related shelf-life was evaluated and compared with Principal Components Regression (PCR). The training set consisted of dynamic headspace gas chromatographic data collected during storage of pasteurized milk (input information for the ANN models used to make a decision) and its corresponding shelf-life (prediction or response). The study revealed that ANN had better predictability than PCR. A standard error of the estimate of 2 days in shelf life resulting from regression analysis of experimental versus predicted values indicated a high predictability of ANN [4]. A methodology was developed for time series sales forecasting for short shelf-life food products based on ANN models and evolutionary computing. The methodology was claimed to be particularly useful for manufacturers of fresh milk, since successful sales forecasting reduces considerably the lost sales and products returns [5].

\subsection{Yogurt}

\subsubsection{Control of expiration date}

The changes in the physical, chemical, and microbiological structure of yogurt determine the storage and shelf life of the product. In a reported study microbial counts and $\mathrm{pH}$ values of yogurt during storage were determined at day 1,7 , and 14. Simultaneously, image processing of yogurt was digitized by using a Machine Vision System (MVS) to determine colour changes during storage, and the obtained data were modeled with neurocomputing models for prediction of shelf life of set-type whole-fat and low-fat yogurts. The ANN models were developed using backpropagation networks with a single hidden layer and sigmoid activation functions [6]. The input variables of the network were $\mathrm{pH}$; total aerobic, yeast, mold, and coliform counts; and colour analysis values measured by the MVS. The output variable was the storage time of the yogurt. The modelling results showed that there was excellent agreement between the experimental data and predicted values, with a high determination coefficient $\left(\mathrm{R}^{2}=0.9996\right)$ showing that the developed model was able to analyze non-linear multivariate data with very good performance, fewer parameters, and shorter calculation time. The model might be an alternative method to control the expiration date of yogurt shown in labeling and provide consumers with a safer food supply. 


\subsubsection{Authenticity of low-fat yogurts}

The growing consumption of low- and reduced-fat dairy products demands routine control of their authenticity by health agencies. The usual analyses of fat in dairy products are very simple laboratory methods. However, they require manipulation and use of reagents of a corrosive nature, such as sulfuric acid, to break the chemical bonds between fat and proteins. Additionally, they generate chemical residues that require an appropriate disposal. The use of an ANN based on simple instrumental analyses, such as $\mathrm{pH}$, color, and hardness (inputs) was proposed for the classification of commercial yogurts in the low- and reduced-fat categories (outputs) [7]. A total of 108 strawberry-flavored yogurts (48 probiotic low-fat, 36 low-fat, and 24 full-fat yogurts) belonging to several commercial brands and from different batches were used in this research. The statistical analysis showed different features for each yogurt category; a database was built and a neural model was trained with the Levenberg-Marquardt algorithm by using the neural network toolbox of the software MATLAB 7.0.1. Validation with unseen data pairs showed that the proposed model was $100 \%$ efficient. Because the instrumental analyses do not require any sample preparation and do not produce any chemical residues, the proposed procedure is a fast and interesting approach for monitoring the authenticity of the products.

\subsubsection{Quantitative determination of protein}

A method has been introduced for quantitative determination of protein content in yogurt samples based on the characteristic absorbance of protein in 1800-1500 $\mathrm{cm}^{-} 1$ spectral region by mid-FTIR spectroscopy and chemometrics. Successive Projection Algorithm (SPA) wavelength selection procedure, coupled with feed forward Back-Propagation Artificial Neural Network (BP-ANN) model was the benefited chemometric technique. Relative Error of Prediction in BP-ANN and SPA-BP-ANN methods for training set was 7.25 and 3.70 respectively. Considering the complexity of the sample, the ANN model was found to be reliable, while the proposed method is rapid and simple, without any sample preparation step [8].

\subsection{Chocolate}

A rapid and non-destructive method has been devised for the characterization of chocolate samples based on diffuse reflectance near-infrared Fourier transform spectroscopy (DRIFTS) and ANNs [9]. This methodology provides a potentially useful alternative to time-consuming chemical methods of analysis. To assess its utility, 36 chocolate samples purchased from the Spanish market were analyzed for the determination of the main nutritional parameters like carbohydrates, fat, proteins, energetic value and cocoa content. Direct triplicate measurements of each sample were carried out by DRIFTS. Cluster hierarchical analysis was used 
for selecting calibration and validation data sets, resulting in a calibration set comprised of 19 samples and a validation data set of 17 samples. As it is common the presence of non-linear effect in reflectance spectroscopy, ANNs was chosen for data pretreatment. The root-mean-square error of prediction (RMSEP) values obtained for carbohydrates, fat, energetic value and cocoa were $1.0 \%(\mathrm{w} / \mathrm{w}), 1.0 \%$ $(\mathrm{w} / \mathrm{w}), 50 \mathrm{~kJ}(100 \mathrm{~g})-1$ and $1.4 \%$, respectively. The mean difference $(\mathrm{dx}-\mathrm{y})$ and standard deviation of mean differences $(\mathrm{sx}-\mathrm{y})$ of the carbohydrates, fat, proteins content, energetic value and cocoa content were 0.9 and $2.4 \%(\mathrm{w} / \mathrm{w}), 0.2$ and $1.0 \%(\mathrm{w} / \mathrm{w}), 9.1$ and $50 \mathrm{~kJ}(100 \mathrm{~g})-1$, and -0.5 and $1.4 \%$, respectively. The maximum relative error for the prediction (QC) of any of these parameters for a new sample did not exceed 5.2\%.

\subsection{Butter}

The seasonal variations of the fatty acids composition of butters were investigated over three seasons during a 12-month study in the protected designation of origin Parmigiano-Reggiano cheese area. Fatty acids were analyzed by GC-FID, and then computed by ANN [10]. Compared with spring and winter, butter manufactured from summer milk creams showed an optimal saturated/unsaturated fatty acids ratio ( -8.89 and $-5.79 \%)$, lower levels of saturated fatty acids $(-2.63$ and $-1.68 \%)$ and higher levels of mono-unsaturated $(+5.50$ and $+3.45 \%)$, poly-unsaturated fatty acids $(+0.65$ and $+0.17 \%)$, and rumenic acid $(+0.55$ and $+3.41 \%)$, while vaccenic acid had lower levels in spring and higher in winter $(-2.94$ and $+2.91 \%)$. ANN models were able to predict the season of production of milk creams, and classify butters obtained from spring and summer milk creams on the basis of the type of feeding.

\subsection{Swiss type cheeses}

$\mathrm{Ni}$ and Gunasekaran observed that a three-layer ANN model is able to predict more accurately than regression equations for the rheological properties of Swiss type cheeses on the basis of their composition [11]. Jimenez-Marquez et al. were of the opinion that prediction of moisture in cheese of commercial production using neural networks models can be used both for research to develop the base of knowledge on production variables and their complex interactions, as well as for the prediction of cheese moisture [12].

\subsection{Processed cheese}

Linear Layer (Train) and Generalized Regression models were developed for predicting the shelf life of processed cheese stored at $7-8^{\circ} \mathrm{C}$ [13]. The experimentally obtained data of the product relating to soluble nitrogen, $\mathrm{pH}$; standard plate count, yeast \& mould count, and spore count were taken as input variables; while the sensory score as output for developing the computational 
intelligence models. The data consisted of 36 experimental observations, which were sub-divided into two disjoint sets, i.e., 30 (80\% of total observations) for training, and 6 (20\% of total observations) for validation. The comparison of the two developed models showed that Generalized Regression model with spread constant as 10 got best simulated with less than 1\% RMSE. The study led to conclusion that computational intelligence models are quite effective in predicting the shelf life of processed cheese.

The Time-delay single layer and multilayer ANN models were suggested for predicting the shelf life of processed cheese stored at $7-8^{\circ} \mathrm{C}$ [14]. The input parameters of the ANN consisted of soluble nitrogen, $\mathrm{pH}$; standard plate count, yeast \& mould count, and spore count. The output parameter was sensory score. The results of the experiments showed excellent correlation between the training data and the validation data with a high Nash - Sutcliffo coefficient and determination coefficient, suggesting that the developed models are able to analyze non-linear multivariate data with excellent performance and shorter calculation time. From the study it was inferred that time-delay ANN models are very good for predicting the shelf life of processed cheese.

In another study, Radial Basis (Exact Fit) and Linear Layer (Design) models were proposed for predicting the shelf life of processed cheese stored at $7-8^{\circ} \mathrm{C}$. The laboratory data of the product relating to body \& texture, aroma \& flavour, moisture, and free fatty acids were used as input variables; and sensory score as output. Different combinations of experiments were tried. From the results it was observed that Radial Basis (Exact Fit) model with spread constant as 20 gave best fit, indicating that the Radial Basis (Exact Fit) ANN models are very effective in predicting the shelf life of processed cheese [15].For predicting the shelf life of processed cheese stored at $30^{\circ} \mathrm{C}$, Time-delay artificial neural network (TDNN) computing models with single and multi layers were developed taking soluble nitrogen, $\mathrm{pH}$; standard plate count, yeast \& mould count, spore count of the product as input variables, and sensory score as output [16].Mean square error, root mean square error, coefficient of determination and Nash - Sutcliffo coefficient were included in the study with the purpose to compare the prediction ability of the developed TDNN models. Regression equations were developed for estimating the shelf life of processed cheese, which came out as 28.25 days as against experimentally determined shelf life of 30 days. The Radial Basis (Exact Fit) and Linear Layer (Design) ANN models were proposed for predicting the shelf life of processed cheese stored at $30^{\circ} \mathrm{C}$ [17]. Soluble nitrogen, $\mathrm{pH}$; standard plate count, yeast $\&$ mould count, and spore count of the product were used as input variables and sensory score as output. Mean square error, root mean square error, coefficient of determination and Nash - Sutcliffo coefficient were applied with the objective to compare the prediction ability of the developed models. Several experiments were carried out and it was observed that Radial Basis (Exact Fit) model with 30 neurons and spread constant as 20 performed the best, hence it was selected for predicting the shelf life of processed cheese, which came out as 28.54 days vis-à-vis 30 days experimental shelf life. From the study it was 
concluded that the developed computing model is quite efficient in predicting the shelf life of processed cheese.

A novel method for shelf life detection of processed cheese using Cascade single and multi layer ANN computing models was recommended for estimating the shelf life of processed cheese stored at $30^{\circ} \mathrm{C}$ [18]. The performance of the different developed models was compared with each other. Cascade backpropagation algorithm (CBA) model with double hidden layer having 27:27 neurons gave most encouraging results. Based on these results regression equations were developed for detecting the shelf life of processed cheese, which came out to be 29.13 days, i.e., quite close to laboratory determined shelf life of 30 days. Therefore, from the study it was inferred that CBA based ANN models are quite effective in detecting the shelf life of processed cheese. The suitability of generalized regression, radial basis (fewer neurons), and linear layer (design) computational ANN techniques for shelf life prediction of processed cheese stored at $30^{\circ} \mathrm{C}$ was evaluated [19]. Generalized Regression model gave better results, hence based on these observations, further regression equations were developed for determining the shelf life of the product, which came out to be 29.49 days which was very near to experimentally obtained shelf life of 30 days. The study revealed that amongst the studied ANN techniques, Generalized Regression method is most effective in predicting the shelf life of processed cheese.

\subsection{Kalakand}

The shelf life of kalakand, which is sweetened milk based desiccated dairy product, was estimated by implementing Cascade single and double hidden layer models. The developed models were compared with each other for observing their supremacy over the other [20]. The network was trained with 100 epochs and number of neurons in single and double hidden layers varied from 1 to 30. Several experiments were carried out with Cascade single and double hidden layers, and various data portioning approaches were used. Different combinations were tried, tested and compared with each other. Cascade models with single hidden layer having four neurons gave the best outcome (MSE 0.000592818; RMSE: $0.024347850 ; \mathrm{R}^{2}: 0.992884381$ ). Cascade models with two hidden layers having twenty neurons in the first layer and twenty neurons in the second layer gave the best fit (MSE 0.000988770; RMSE: 0.03144471; $\mathrm{R}^{2}: 0.988125331$ ) for predicting the shelf life of kalakand stored at $6^{\circ} \mathrm{C}$.

\subsection{Milky White Dessert Jeweled with Pistachio}

The ANNs were applied for predicting the shelf life of milky white dessert jeweled with pistachio [21]. Linear layer (train) and generalized regression models were developed and compared with each other. Neurons in each hidden layers varied from 1 to 30. Data samples were divided into two subsets, i.e., $80 \%$ for training the network and $20 \%$ for validation. Mean square error, root mean square 
error, coefficient of determination and Nash - Sutcliffo coefficient were included in the investigation for comparing the prediction performance of the developed models. The results of the study established that ANNs are effective tool for determining the shelf life of milky white dessert jeweled with pistachios.

\subsection{Burfi}

Radial basis (exact fit) model was suggested for estimating the shelf life of an extremely popular milk based sweetmeat namely burfi [22]. The input variables were the lab data of the product relating to moisture, titratable acidity, free fatty acids, tyrosine, and peroxide value; and the overall acceptability score was output. Mean square error, root mean square error, coefficient of determination and Nash-Sutcliffo coefficient were applied for comparing the prediction ability of the developed models. The observations indicated exceedingly well correlation between the actual data and the predicted values, with a high determination coefficient and Nash-Sutcliffo coefficient establishing that the models were able to analyze non-linear multivariate data with very good performance and shorter calculation time. From the study it was concluded that the developed model, which is very convenient, less expensive and less time consuming, can be a good alternative to expensive, time consuming and cumbersome laboratory testing method for estimating the shelf life of burfi.

\subsection{Cakes}

Goyal and Goyal implemented brain based artificial intelligent scientific computing models for shelf life detection of cakes stored at $30^{\circ} \mathrm{C}$. Cascade Neural Network (CNN) and Probabilistic Neural Network (PNN) models were developed [23]. Input variables for ANNs were the experimental data of the product pertaining to moisture, titratable acidity, free fatty acids, peroxide value, and tyrosine; while overall acceptability score was output variable. Mean Square Error, Root Mean Square Error, Coefficient of determination and Nash - Sutcliffo Coefficient were included in the study with the aim to compare the prediction performance of the developed models. The data samples consisted of 60 observations, which were divided in two disjoint subsets, viz., training set having 48 observations and validation set 12 . The best results of CNN with single hidden layer having twenty five neurons were MSE: 0.000356548, RMSE: 0.018882467, $\mathrm{R}^{2}: 0.995721429, \mathrm{E}^{2}: 0.999643452$; and with two hidden layers having nine neurons in the first and second layer were MSE: 0.000364059, RMSE: $0.019080335, \mathrm{R}^{2}: 0.99563129, \mathrm{E}^{2}: 0.999635941$.The results for PNN were MSE: 0.000582052; RMSE: 0.024125759; $\mathrm{R}^{2}: 0.993015373 ; \mathrm{E}^{2}$ : 0.999417948 . The best results of all the models were compared with each other, and it was observed that $\mathrm{CNN}$ model with single hidden layer having twenty five neurons gave the best fit for shelf life estimation of cakes. 


\subsection{Soft Cakes}

The potential of simulated neural networks $(\mathrm{SNN})$ for predicting the shelf life of soft cakes stored at $10^{\circ} \mathrm{C}$ was highlighted by Goyal and Goyal [24]. Elman and self organizing SNN models were developed. In this study, the lab data of the product related to moisture, titratable acidity, free fatty acids, tyrosine, and peroxide value were input variables; and overall acceptability score was output. Neurons in each hidden layers varied from 1 to 30 . The network was trained with single as well as double hidden layers with 1500 epochs. The transfer function for hidden layer was tangent sigmoid; while for the output layer, it was pure linear. The experiments revealed that SNN models are excellent tool for predicting the shelf life of soft cakes.

\subsection{Brown Milk Cakes}

The shelf life of brown milk cakes decorated with almonds was determined by ANN based radial basis (exact fit) and radial basis (fewer neurons) models, and the developed models were compared with each other. Both the developed models predicted the shelf life of the product exceedingly well [25]. However, comparison of the developed models gave very interesting observation, i.e., output results were the same when numerous experiments were conducted after having taken the same spread constant in both the models. The study revealed that both the models are convenient and powerful alternative tool to laboratory's cumbersome, expensive and long time taking shelf life testing method for predicting the shelf life of brown milk cakes decorated with almonds.

\subsection{Soft Mouth Melting Milk Cakes}

The time-delay and linear layer (design) intelligent computing expert system models were proposed for predicting the shelf life of soft mouth melting milk cakes stored at $6^{\circ} \mathrm{C}$. The best results for time-delay model with single hidden layer having 20 neurons were observed with MSE: 0.001332342, RMSE: 0.036501259,

$\mathrm{R}^{2}$ : 0.984011897; and for double hidden layers having 8 neurons in the first and second layers with MSE: 0.001318004, RMSE: 0.036304329, R²: 0.984183948 , while best results for linear layer (design) model were with MSE: 0.000293366, RMSE: $0.017127919, \mathrm{R}^{2}$ : 0.996479613 . The outcome of the study established that intelligent computing expert system models are quite effective in predicting the shelf life of soft mouth melting milk cakes [26].Central Nervous System (CNS) based intelligent computer engineering system were recommended to detect the shelf life of soft mouth melting milk cakes stored at $10^{\circ} \mathrm{C}$. Feedforward backpropagation single layer and multilayer models along with linear layer (design) models were developed and compared with each other [27]. The dataset was randomly divided into two disjoint subsets, viz., training set having $80 \%$ of the total observations and testing set $20 \%$ of the total observations. For 
developing the CNS intelligent systems, input variables were the experimental data of the product relating to moisture, titratable acidity, free fatty acids, peroxide value, and tyrosine; while overall acceptability score output. Number of neurons in each hidden layer varied from 1 to 30. The network was trained with 1000 epochs. Mean square error, root mean square error, coefficient of determination and Nash - Sutcliffo coefficient were used for comparing the prediction ability of the developed models. The researchers observed that feedforward model with single hidden layer having twenty eight neurons gave the best result. Based on these results regression equations were developed for predicting the shelf life, which came out as 19.82 days vis-à-vis 21 days experimental shelf life. From the study it was concluded that CNS based intelligent computer engineering system are quite efficient in forecasting the shelf life of soft mouth melting milk cakes.

\subsection{Instant Coffee Drink}

For forecasting the shelf life of instant coffee drink, radial basis artificial neural engineering and multiple linear regression models have been developed by using the experimental data of the product relating to colour and appearance, flavour, viscosity and sediment as input variables; and overall acceptability score as the output. The study showed that the multiple linear regression model was superior to radial basis model for determining the shelf life of the product [28].

\subsection{Instant coffee flavoured sterilized drink}

Goyal and Goyal developed Cascade forward and feedforward backpropagation artificial intelligence models for predicting the sensory quality of instant coffee flavoured sterilized drink. Different combinations of several internal parameters, i.e. data pre-processing, data partitioning, number of hidden layers, number of neurons in each hidden layer, transfer function, error goal, etc., along with backpropagation algorithm based on Levenberg-Marquardt mechanism as training function were explored. The network was trained with 100 epochs, and the number of neurons in each hidden layer varied from 1 to 20 . The results of the two models were evaluated with three types of prediction performance measures, viz., Root mean square error, coefficient of determination $\mathrm{R}^{2}$ and mean square error. Feedforward backpropagation artificial intelligence model exhibited best results (3.70\% RMSE; $0.998 \mathrm{R}^{2}$; $\left.0.0013 \mathrm{MSE}\right)$, followed by Cascade forward artificial intelligence model (5.36\% RMSE; $0.996 \mathrm{R}^{2}$; $\left.0.0028 \mathrm{MSE}\right)$. The study showed that the feedforward backpropagation model was better than cascade forward artificial intelligence model for estimating the sensory quality of instant coffee flavoured sterilized drink [29].Elman and generalized regression artificial intelligence models for detecting the shelf life of instant coffee flavoured sterilized drink have also been proposed. In this research the lab data of the product concerning colour and appearance, flavour, viscosity, sediment were input variables; and the overall acceptability score output variable. The dataset 
consisted of 50 observations, which were randomly divided into two disjoint subsets, viz., training set consisting of 40 observations and validation set having 10 observations. Mean square error and root mean square error were used as prediction performance measures. The best result for Elman models were: Neurons 4:4; MSE: 0.001101304; RMSE: 0.033185894; and for generalized regression models, Spread Constant: 2; MSE: 0.164212158; RMSE: 0.405230993. Between the two developed models, Elman artificial intelligence models demonstrated a better approach [30].

\subsection{Roasted Coffee Flavoured Sterilized Drink}

Cascade single and double layer ANN models for estimating the sensory quality of roasted coffee flavoured sterilized drink have been suggested by applying the experimental data of the product relating to colour and appearance, viscosity, flavour and sediment as input variables; and overall acceptability score as the output [31].The results of the cascade ANN models were calculated with two types of prediction performance measures, viz., root mean square error and coefficient of determination $\mathrm{R}^{2}$. The study revealed that more the number of neurons in single hidden layer, less is the error for cascade ANN models ( RMSE:0.00011; R² : 0.999999; neurons:50).

\subsection{Post-Harvest Roasted Coffee Sterilized Milk Drink}

Artificial intelligence neural network Elman model was proposed for predicting the shelf life of roasted coffee sterilized cow milk drink stored at $30^{\circ} \mathrm{C}$. In order to compare the prediction potential of the Elman model, Radial Basis model was also developed [32]. The final results of both the models were compared with each other. The Elman model with single hidden layer having eighteen neurons gave the best fit (MSE: 9.97756E-07, RMSE: 0.000998877, $\mathrm{R}^{2:} 0.999990022, \mathrm{E}^{2 \text { : }}$ 0.999996211), followed by Elman model with two hidden layers having 7 neurons in the first layer and 5 in the second layer ( MSE: 8.48661E-06, RMSE : $\left.0.002913179, \mathrm{R}^{2}: 0.999915134, \mathrm{E}^{2}: 0.999999923\right)$; and Radial Basis model with spread constant as 100 (MSE : 4.1554E-05, RMSE: 0.006446238, $\mathrm{R}^{2}$ : $\left.0.99958446, E^{2}: 0.999951677\right)$, respectively. From the study it was concluded that artificial intelligence models are quite effective in predicting the shelf life of the product. Regression equations were developed to quantify the shelf life, which was found to be 37.80 days vis-à-vis 45 days experimentally obtained shelf life. The authors were of the view that since the predicted value was within the experimentally determined value, hence the product should be accepted. 


\section{Conclusion}

The application of ANNs for predicting the shelf life of food products in food industry is quite a new and effective approach. The ANN technique for food products was applied mainly because the shelf life evaluation in the laboratory is very cumbersome, expensive, and time-consuming, while the ANN procedure is sensitive, reliable, fast, simple and low-cost method for monitoring the authenticity of the products, which provide consumers with a safer food supply. This review discusses the systematic information available in the literature concerning the application of ANN models for shelf life determination of milk, various dairy products, viz., yoghurt, chocolate, butter, cheese, kalakand, dairy confections and milk based coffee drinks. The information provided in this communication would be very useful for the dairy industry, consumers, wholesalers, retailers, regulatory agencies, food researchers and academicians.

\section{References}

[1] Krogh, A. (2008). What are artificial neural networks?. Nature Biotechnology, 26(2), 195-197.

[2] Chayjan, R.A. (2010). Modeling of sesame seed dehydration energy requirements by a soft- computing. Australian Journal of Crop Science, 4(3), 180-184.

[3] www.medlabs.com/Downloads/food_product_shelf_life_web.pdf (accessed on 11.5.2011).

[4] Vallejo-Cordoba, B., Arteaga, G. E. and Nakai, S. (1995). Predicting milk shelf-life based on artificial neural networks and headspace gas chromatographic data. Journal of Food Science, 60, 885-888.

[5] Doganis, P., Alexandridis, A., Patrinos, P. and Sarimveis, H. (2006). Time series sales forecasting for short shelf-life food products based on artificial neural network models and evolutionary computing. Journal of Food Engineering, 75,196-204.

[6] Sofu, A and Ekinci, F.Y. (2007). Estimation of Storage Time of Yogurt with Artificial Neural Network Modeling. Journal of Dairy Science, 90(7), 31183125.

[7] Cruz.A.G., Walter, E.H.M., Cadena, R.S., Faria, J.A.F., Bolini, H.M.A. and Fileti, A.M.F. (2009). Monitoring the authenticity of low-fat yogurts by an artificial neural network. Journal of Dairy Science, 92(10), 4797-4804.

[8] Khanmohammadi, M., Garmarudi, A.B., Ghasemi, K., Garrigues, S. and Guardia, M. (2009). Artificial neural network for quantitative determination of total protein in yogurt by infrared spectrometry. Microchemical Journal, $91(1), 47-52$.

[9] Moros, J., Iñón, F.A., Garrigues, S. and Guardia, M. (2007). Near-infrared diffuse reflectance spectroscopy and neural networks for measuring 
nutritional parameters in chocolate samples. Analytica Chimica Acta, 584(1), 215-222.

[10] Gori, A., Chiara, C., Selenia, M., Nocetti, M., Fabbri, A., Caboni, M. F. and Losi, G. (2011). Prediction of seasonal variation of butters by computing the fatty acids composition with artificial neural networks. European Journal of Lipid Science and Technology, 113(11), 1412-1419.

[11] Ni, H. and Gunasekaran, S. (1998). Food quality predication with neural networks. Food Technology, 52 (10), 60-65.

[12] Jimenez-Marquez, S. A., Thibault, J. and Lacroix, C. (2005). Prediction of moisture in cheese of commercial production using neurocomputing models. International Dairy Journal, 15, 1156-1174.

[13] Goyal, Sumit and Goyal, G.K. (2012). Linear layer and generalized regression computational intelligence models for predicting shelf life of processed cheese. Russian Journal of Agricultural and Socio-Economic Sciences, 3(3), 28-32.

[14] Goyal, Sumit and Goyal, G.K. (2012). Shelf life estimation of processed cheese by artificial neural network expert systems. Journal of Advanced Computer Science and Technology, 1(1), 32-41.

[15] Goyal, Sumit and Goyal, G.K. (2012). Radial basis (exact fit) and linear layer (design) computerized ANN models for predicting shelf life of processed cheese. Computer Science Journal, 2(1), 11-18.

[16] Goyal, Sumit and Goyal, G.K. (2012). Time-Delay artificial neural network computing models for predicting shelf life of processed cheese. BRAIN. Broad Research in Artificial Intelligence and Neuroscience, 3(1), 63-70.

[17] Goyal, Sumit and Goyal, G.K. (2012). Radial basis (exact fit) and linear layer (design) ANN models for shelf life prediction of processed cheese. International Journal of $u$ - and e- Service, Science and Technology, 5(1), 6369.

[18] Goyal, Sumit and Goyal, G.K. (2012). A novel method for shelf life detection of processed cheese using cascade single and multi layer artificial neural network computing models. ARPN Journal of Systems and Software, 2(2), 79-83.

[19] Goyal, Sumit and Goyal, G.K. (2011). Performance of generalized regression, radial basis (fewer neurons), and linear layer (design) computational ANN techniques for shelf life prediction of processed cheese. International Journal of Artificial Intelligence and Knowledge Discovery, $1(4), 12-15$.

[20] Goyal, Sumit and Goyal, G.K. (2011). Advanced computing research on cascade single and double hidden layers for detecting shelf life of kalakand: An artificial neural network approach. International Journal of Computer Science and Emerging Technologies, 2(5), 292-295.

[21] Goyal, Sumit and Goyal, G.K. (2011). A new scientific approach of intelligent artificial neural network engineering for predicting shelf life of 
milky white dessert jeweled with pistachio. International Journal of Scientific and Engineering Research, 2(9), 1-4.

[22] Goyal, Sumit and Goyal, G.K. (2012). Radial basis (exact fit) artificial neural network technique for estimating shelf life of burfi. Advances in Computer Science and its Applications, 1(2), 93-96.

[23] Goyal, Sumit and Goyal, G.K. (2011). Brain based artificial neural network scientific computing models for shelf life prediction of cakes. Canadian Journal on Artificial Intelligence, Machine Learning and Pattern Recognition, 2(6), 73-77.

[24] Goyal, Sumit and Goyal, G.K. (2011). Simulated neural network intelligent computing models for predicting shelf life of soft cakes. Global Journal of Computer Science and Technology, 11(14), Version 1.0, 29-33.

[25] Goyal, Sumit and Goyal, G.K. (2011). Radial basis artificial neural network computer engineering approach for predicting shelf life of brown milk cakes decorated with almonds. International Journal of Latest Trends in Computing, 2(3), 434-438.

[26] Goyal, Sumit and Goyal, G.K. (2011). Development of intelligent computing expert system models for shelf life prediction of soft mouth melting milk cakes. International Journal of Computer Applications, 25(9), 41-44.

[27] Goyal, Sumit and Goyal, G.K. (2012). Central nervous system based computing models for shelf life prediction of soft mouth melting milk cakes. International Journal of Information Technology and Computer Science, 4(4), 33-39.

[28] Goyal, Sumit and Goyal, G.K. (2011).Application of artificial neural engineering and regression models for forecasting shelf life of instant coffee drink. International Journal of Computer Science Issues, 8(4), No. 1, 320324.

[29] Goyal, Sumit and Goyal, G.K. (2011).Cascade and feedforward backpropagation artificial neural networks models for prediction of sensory quality of instant coffee flavoured sterilized drink. Canadian Journal on Artificial Intelligence, Machine Learning and Pattern Recognition, 2(6), 7882.

[30] Goyal, Sumit and Goyal, G.K. (2011). Development of neuron based artificial intelligent scientific computer engineering models for estimating shelf life of instant coffee sterilized drink. International Journal of Computational Intelligence and Information Security, 2(7), 4-12.

[31] Goyal, Sumit and Goyal, G.K. (2012). Study on single and double hidden layers of cascade artificial neural intelligence neurocomputing models for predicting sensory quality of roasted coffee flavoured sterilized drink. International Journal of Applied Information Systems, 1(3), 1-4.

[32] Goyal, Sumit and Goyal, G.K. (2011). Computerized models for shelf life prediction of post-harvest coffee sterilized milk drink. Libyan Agriculture Research Center Journal International, 2 (6), 274-278. 